\title{
First Demonstration of an Amplified Transmission Line Based on Multi-Element Fibre Technology
}

\author{
V. J. F. Rancaño*, S. Jain, T. C. May-Smith, J. K. Sahu, P. Petropoulos, D. J. Richardson \\ Optoelectronics Research Centre, University of Southampton, UK \\ vjfr1u10@orc.soton.ac.uk
}

\begin{abstract}
We report the first amplified transmission experiments based on multi-element transmission fibres and amplifiers. The system exhibits negligible levels of crosstalk and compatibility with traditional WDM systems. The technology allows access to individual fibre elements without the need for any special interfacing devices.
\end{abstract}

\section{Introduction}

The capacity limits of standard single mode fibre systems are within sight and recent traffic growth estimations ${ }^{1,2}$ imply that the trend of ever decreasing cost-per-bit as commercial system capacities are increased will soon reach a turning point since the only way to absorb the additional traffic will be to install additional parallel single mode WDM systems. Such unfavourable economics have the potential to constrain future growth of the internet.

Space Division Multiplexing (SDM) offers a possible route to improve the economics of installing and operating future ultrahigh capacity networks. The underlying premise is that cost of performing key functions such as amplification and routing for an $\mathrm{N}$ spatial-channel SDM system should be cheaper than for $\mathrm{N}$ single mode systems of equivalent total capacity due to the greater spatial proximity of channels and the potential component sharing and device integration that this offers. The main approaches to implementing SDM have so far been based on either multicore (MCF) or multimode fibres (MMF).

$\mathrm{MCFs}^{3}$ allow parallel data transmission through a single fibre with several effectively independent cores. Mechanical reliability considerations however limit the maximum outer diameter (OD) to around $225 \mu \mathrm{m}^{4}$, and the need to restrict inter core cross-talk limits the maximum number of cores to $\sim 13^{5}$.

$\mathrm{MMFs}^{6}$ allow parallel transmission channels defined by the different propagation modes. The inherent mode coupling must be compensated electronically using multiple-input multiple-output (MIMO) processing leading to considerable increases in system complexity ${ }^{7}$, as well as increased power consumption and cost which need to be offset by savings elsewhere in the system to make the approach competitive commercially. In addition, both MCF and MMF approaches require the development of specialised components for coupling to/from the individual SDM channels ${ }^{8-11}$.

Many of the limitations associated with MCF and MMF systems can be overcome by employing the SDM technology studied in this work, and which is based on multi-element fibres (MEFs). MEFs can be viewed as a bundle of independent fibres (elements) drawn together into a common polymer coating: each element comprising an independent core and cladding ${ }^{12}$. In principle, as opposed to the MCF, there is no limit to the diameter of an MEF (and associated number of elements) provided that the mechanical robustness of the individual elements is maintained. In addition, the MEF geometry ensures that there is no crosstalk between the individual elements. Moreover, access to each element is gained simply by stripping off the coating, thereby ensuring this type of fibre is readily compatible with standard single mode fibres (SMFs). MEF technology can also be easily extended to the manufacture of multi-element erbium doped fibre amplifiers (ME-EDFA). In fact, as will be shown here, cladding-pumped ME-EDFAs are readily realised which is critical in lowering the cost per transmitted bit.

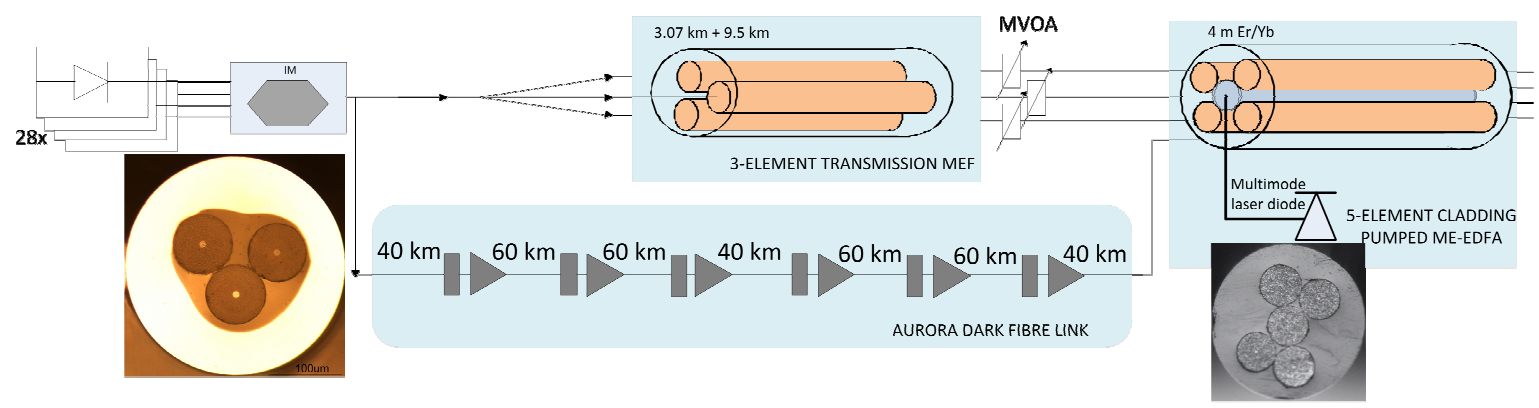

Fig. 1 Experimental Set-up and cross-section of the MEF ${ }^{12}$ and ME-EDFA ${ }^{13}$ fibres 
In this paper, we report the first amplified transmission experiments using MEFs and a cladding-pumped ME-EDFA. Our experiments show that MEF technology is fully compatible with standard installed SMF systems and that the channels can be considered truly independent.

\section{Transmission experiments}

We set up a transmission experiment emulating a hypothetical scenario of a smooth system upgrade from a single-fibre WDM system to an MEF-based SDM one. The experimental setup is shown in Fig. 1. The transmitter consisted of 28 channels in the range $1535 \mathrm{~nm}$ to $1562 \mathrm{~nm}$, modulated using a $10 \mathrm{Gbit} / \mathrm{s} 2^{31}-1 \mathrm{NRZ}$ OOK pseudorandom bit sequence. The transmission line comprised (a) a cascade of two 3-element MEFs with the features described in ref. 12 (see Fig.1(inset) for an image of the fibre crosssection) and respective lengths of $3.07 \mathrm{~km}$ and $9.5 \mathrm{~km}$ and (b) a WDM $400 \mathrm{~km}$ installed dark fibre link (part of the UK's AURORA network). The MEFs were cascaded using standard SMF connectors that were spliced to each element of the MEF using a standard splicer. A 5-element cladding-pumped ME-EDFA, comprising one central (un-doped) multimode element for pump delivery and coupling and surrounded by $4 \mathrm{Er}$ $\mathrm{Yb}$ doped signal-elements ${ }^{13}$ (see Fig.1(inset)), was used at the output of the transmission line. The three elements of the MEF were connected to three of the input ports of the ME-EDFA, the fourth port of which was connected to the dark fibre link. Three manual variable optical attenuators (MVOA) were installed at the input ports of the ME-EDFA to ensure that the net loss of each transmission path was $0 \mathrm{~dB}$.

Fig.2a presents the gain and noise figure (NF) characterisation for each of the elements of the ME-EDFA at a pump power of $6.4 \mathrm{~W}$ and an input signal power of $-23 \mathrm{dBm}$. A minimum NF of $4.7 \mathrm{~dB}$ with a maximum gain of about $34 \mathrm{~dB}$ was observed per fibre element. In interpreting these results, it is to be noted that our current MEEDFA does not include any isolators or gain flattening elements and we have yet to optimize its length. The ME-EDFA exhibits a high absorption at $1530 \mathrm{~nm}$ due to a high $\mathrm{Er}-\mathrm{Yb}$ concentration. This feature, together with the nature of cladding-pumping, provides flatter and less noisy gain at wavelengths near the L-band. We therefore anticipate that improved results should be obtained by using a longer amplifier length and operating the system at longer wavelengths. We also anticipate that improvements in fibre fabrication should allow for improvements in uniformity of amplifier element performance.
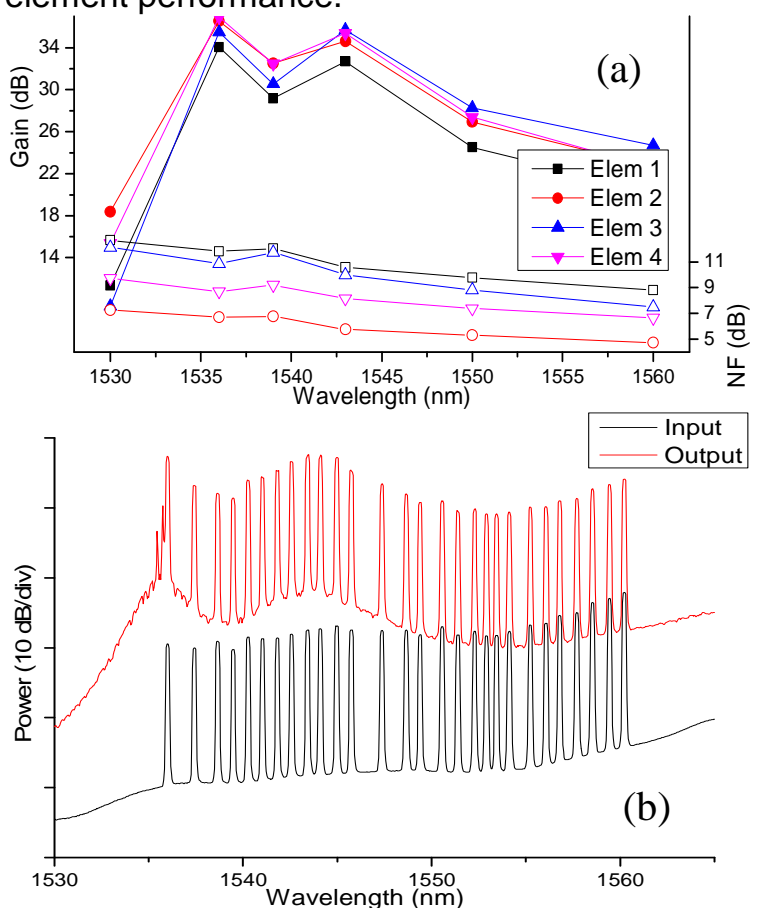

Fig. 2 Gain and noise figure of the elements of the ME-EDFA (top). Input and output spectrum for the signals coming from the dark fibre link (bottom)

Fig. $2 \mathrm{~b}$ presents the spectrum at the input and the output of the ME-EDFA for the signal transmitted through the dark fibre link. The BER of all channels propagating through the four elements of the ME-EDFA was assessed at the output of the amplifier and error-free transmission $\left(B E R<10^{-9}\right)$ was verified for both the MEF and the dark fibre paths. An example of the BER performance of the system including the ME-EDFA for both types of paths is displayed in Fig.3.

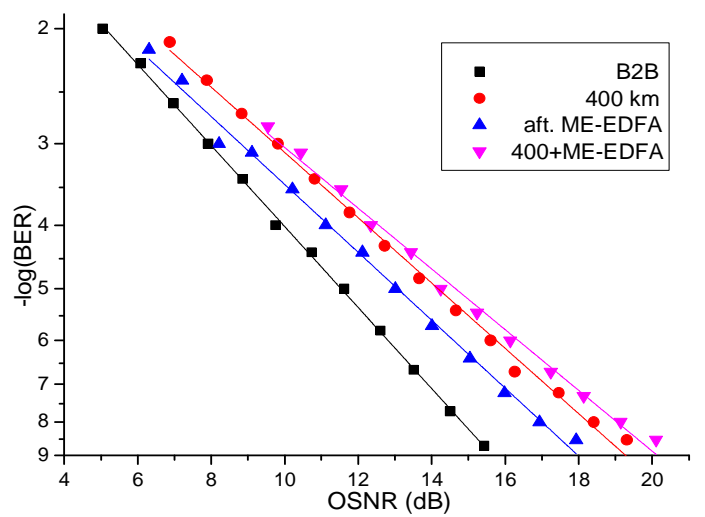

Fig. 3 BER curves for the single amplification experiment

It has been shown previously ${ }^{12}$ that one of the important features of MEF technology is that MEFs are virtually immune to crosstalk between elements. In order to verify that no crosstalk arises in the ME-EDFA either, we characterised 
the signals at each of the ME-EDFA output ports while the remaining fibre amplifier elements were either loaded with the data signals or not. By adjusting the OSNR of the signals traversing one element of the ME-EDFA, so that the BER at the output was $10^{-8}$, we measured the deviation from this BER value while successively turning on the signals in the remaining fibre elements. .No change in the detected signal power and no measurable degradation in the BER of any of the assessed channels were observed irrespective of the number of elements of the ME-EDFA used in parallel and their input powers, thus confirming that the various elements were completely independent in terms of crosstalk, and/or crossgain modulation effects.
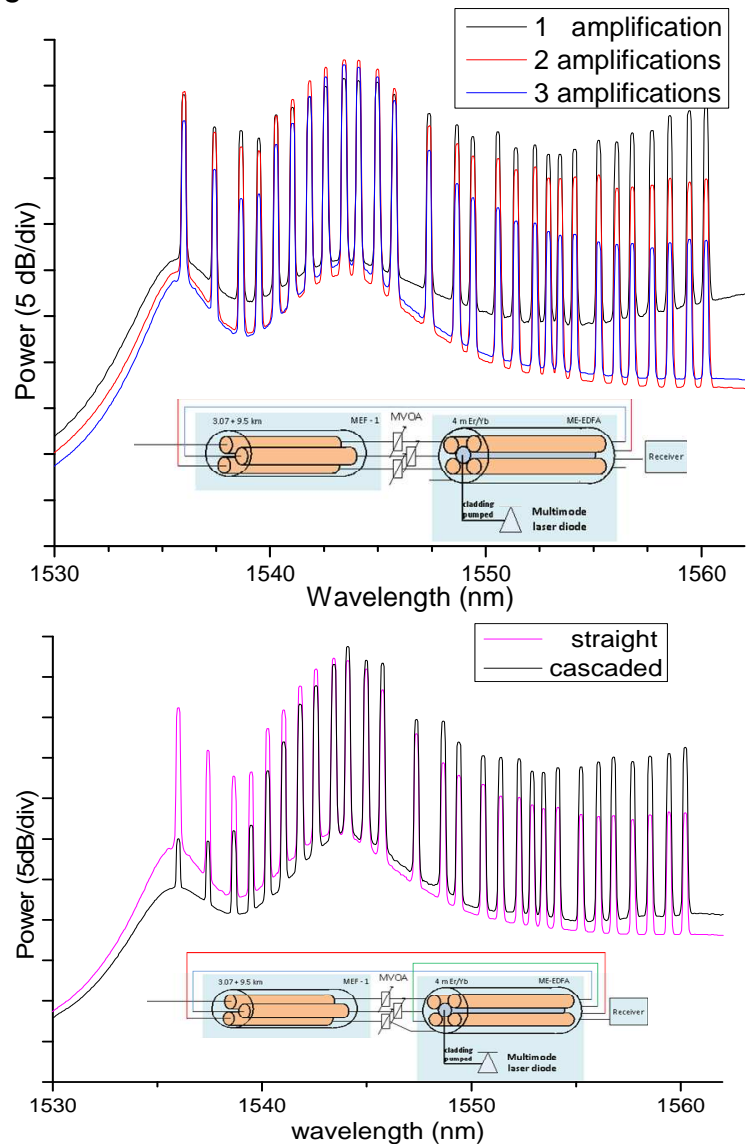

Fig. 4 Spectra after three amplifications with three single-stage ME-EDFA (top) and with two single stage and a dual-stage amplifier (bottom).

In order to assess the performance of a chain of ME-EDFAs, we next connected the elements of the MEFs in succession and used amplifying elements of the ME-EDFA between them (see inset to Fig.4a). The accumulated spectral gain ripple of the ME-EDFA led to gain variation of up to $15 \mathrm{~dB}$ between the transmitted wavelength channels leading to compromised data transmission at wavelengths beyond $1545 \mathrm{~nm}$. In order to demonstrate the sort of improved performance that might be achieved by optimising the amplifier length, we modified the set-up of Fig. 4a; the second stage of amplification was made up by cascading two elements of the ME-EDFA. The result is presented in Fig.4b, which shows a more equalised performance at longer wavelengths. We verified that by using this configuration it was possible to achieve error-free performance for all channels beyond $1540 \mathrm{~nm}$, indicating that important performance benefits are to be gained from an optimised amplifier design.

\section{Conclusions}

We have experimentally demonstrated the first implementation of an amplified MEF system. Both multi-element transmission fibre and EDFA exhibit crosstalk-free operation, full compatibility with existing WDM systems and enough flexibility to be considered as a candidate for fully functional SDM systems. We have included for the first time a cladding-pumped ME-EDFA in a transmission system, and have shown that with suitable design, its gain profile can be effectively shaped for optimised operation in SDM-WDM systems.

\section{Acknowledgements}

This work was supported by the EPSRC grant EP/I01196X: Transforming the Future Internet: The Photonics Hyperhighway. The authors thank JANET, the UK's research and education network, for providing the installed fibre link used during these experiments.

\section{References}

[1] D.J.Richardson, Science. 330, 327 (2010).

[2] R.W.Tkach, Bell Labs Tech. J. 14, 3 (2010)

[3] Y.Awaji et al., Optical Fiber Telecom. VI-B, chap. 13, Elsevier (2013)

[4] K.Imamura et al., ECOC, P1.09 (2010)

[5] J.Sakaguchi et al., OFC PDP5C.1 (2012)

[6] D.W.Peckham et al., Optical Fiber Telecom. VI-A, chap. 8, Elsevier (2013)

[7] K.-P.Ho and J.M.Kahn, JLT 29, 3119 (2011)

[8] B.Zhu et al., Opt. Express. 19, 16665 (2011)

[9] R.R.Thomson et al., Opt. Express. 15, 11691 (2007)

[10] W.Q.Thornburg et al., Opt. Lett. 19(7), 454 (1994)

[11] S.G. Leon-Saval et al., Opt. Exp. 18(8), 8430 (2010)

[12] S.Jain et al., ECOC MO.4.A.2 (2013)

[13] S. Jain et al., Workshop on Specialty Optical

Fibers and their Applications W5.4 (2013). 